\title{
Cognitive and mnemonic dysfunctions in rats with hippocampal-entorhinal lesions: Attenuating effects of glycine injections
}

\author{
TROND MYHRER and TONE STRØMME JOHANNESEN \\ Norwegian Defence Research Establishment, Division for Environmental Toxicology, Kjeller, Norway
}

\begin{abstract}
Transections of the fiber connections between the temporal cortex (TC) and the lateral entorhinal cortex (LEC) result in decreased concentration of glutamate in TC and LEC, and also result in impaired memory. Administration of glutamatergic agonists such as glycine restores mnemonic function in rats with TC/LEC lesions. Because TC/LEC transections combined with damage to the predominantly glutamatergic hippocampus (Hipp) can severely impede both the acquisition and retention of a discrimination task, it appeared pertinent to examine the impact of glycine on rats with combined lesions. The results from Experiment 1 show that one injection of glycine made rats with Hipp + TC/LEC lesions indistinguishable from controls in acquiring and retaining the discrimination task. The complete restoration of functions may be associated with the incomplete lesions used in the combination. To highlight this issue, rats with total hippocampal lesions were treated with the same procedure (Experiment 2). The results show that glycinetreated rats displayed only some improvement in learning the task. The findings are discussed in terms of the localization of mitigating processes and the potential clinical relevance of agonists that enhance glutamatergic neurotransmission.
\end{abstract}

The temporal-hippocampal region has been shown to be very important for learning and memory in rodents and nonhuman primates (see Myhrer, 1992; Squire, 1992). In the rat, lesions encompassing the hippocampus proper, fascia dentata, and subiculum (together denoted as the hippocampal region in this study) only impede acquisition of a discrimination task, whereas damage to the entorhinal cortex only impedes retention of the same task (Myhrer, 1992). These findings have led to the suggestion that the hippocampal region and the entorhinal cortex are differently involved in information processing and storing (Myhrer, 1992).

The hippocampal region receives sensory information from neocortical association areas by way of the perirhinal and entorhinal cortices. This information is transmitted from the medial entorhinal cortex (MEC) and the lateral entorhinal cortex (LEC) via the perforant path (PP) projection system to the hippocampal region. In return, the hippocampal region is able to send information to the entire cortical mantle. Furthermore, LEC is heavily connected with the temporal cortex (TC), whereas the connections between TC and MEC are relatively modest. The fiber connections of TC and LEC are routed in the adjacent white matter (see Myhrer, 1992).

Results from a recent study of proactive memory in the rat show that hippocampal or PP lesions impede acquisition of a brightness discrimination task but not retention

This research was supported by Research Council of Norway Grant 326.93/010 to Trond Myhrer. Correspondence should be addressed to T. Myhrer, Norwegian Defence Research Establishment, Division for Environmental Toxicology, P.O. Box 25, N-2007 Kjeller, Norway. of this task. Moreover, neither TC nor LEC lesions impede acquisition, and only LEC lesions impede retention. However, transection of the connections between TC and LEC impedes both acquisition and retention (Myhrer, 1992). In a subsequent study examining potential effects of combining lesions critical for cognitive functions, it was found that hippocampal lesions combined with either LEC lesions or TC/LEC transections impede both acquisition and retention of the discrimination task (Myhrer \& Johannesen, 1993).

Neurotransmission in the temporal and hippocampal regions is predominantly glutamatergic (Fonnum, 1984; Köhler, 1986). TC/LEC transections are accompanied by reduced concentration of glutamate in both TC and LEC (Myhrer, Iversen, \& Fonnum, 1989). Administration of the glutamatergic agonist glycine restores both proactive and retroactive memory in rats bearing TC/LEC lesions (Myhrer, Johannesen, \& Spikkerud, 1993; Myhrer \& Paulsen, 1992). The present study was designed to extend these previous findings by administering glycine to rats with combined hippocampal and TC/LEC lesions.

The allosteric agonist glycine crosses the blood-brain barrier very slightly, but administration of large doses of glycine do raise the brain levels of glycine (Toth \& Lajtha, 1981). Glycine acts on at least two distinct populations of receptors: the strychnine-sensitive receptor, mostly found in the brain stem and spinal cord, and the strychnine-insensitive NMDA receptor complex in the forebrain. The marked mitigating effects seen in glycinetreated TC/LEC animals most likely activated the glycine site at the NMDA receptors (Myhrer \& Paulsen, 1992). Since the behavioral effects outlasted the pharmacologi- 
cal ones, glycine probably acts through long-term potentiation (LTP). Thus, the purpose of the present study was to determine whether glycine might also restore cognitive functions in rats when hippocampal lesions are combined with TC/LEC transections. Simultaneous damage to several structures, which are all critically involved in learning and memory, may reduce the possibility of improving functions by means of pharmacological agents. It is of interest to investigate the pharmacological impact of glutamatergic agonists because they may be of clinical importance. Various groups of individuals with impaired cognitive functions may profit from relief measures that increase glutamatergic neurotransmission. It has, for instance, been suggested that patients with Alzheimer's disease suffer from glutamatergic dysfunctions in the temporal region (Deutsch \& Morihisa, 1988; Myhrer, 1993). Administration of glycine is believed to facilitate transmission by endogenous glutamate at the NMDA receptor complex in residual neuronal systems. A single dose of glycine $(750 \mathrm{mg} / \mathrm{kg}$ ) was injected just prior to acquisition of the brightness discrimination task used in previous studies.

\section{EXPERIMENT 1}

\begin{abstract}
Method
Subjects. Twenty-four male Wistar rats from a commercial supplier (Møllegaard Breeding Laboratories, Denmark), weighing 290-320 $\mathrm{g}$ at the time of surgery, served as subjects. They were randomly assigned to three groups: Eight rats received combined bilateral hippocampal and TC/LEC lesions and were injected with glycine (the Hipp + TC/LEC + Gly group), 8 rats received combined bilateral Hipp and TC/LEC lesions and were injected with saline (the Hipp + TC/LEC + Sal group), and 8 sham-operated rats served as the control group. The rats were housed individually and had free access to commercial rat pellets and water. The rats were handled individually 3 days preoperatively and 1 day postoperatively, and were allowed to explore a table top $(80 \times 60 \mathrm{~cm})$ for 3 min a day. The climatized $\left(21^{\circ} \mathrm{C}\right)$ vivarium was illuminated from 0700 to 1900 hours.
\end{abstract}

Surgery. The rats were anesthetized i.p. with diazepam $(10 \mathrm{mg} / \mathrm{kg})$ and fenatyl fluanisone $(2 \mathrm{mg} / \mathrm{kg}$ ) and placed in a stereotaxic headholder with their skulls horizontal. Bilateral hippocampal lesions were made by aspiration by means of a syringe with cannula (21 gauge, diam $0.8 \mathrm{~mm}$ ) connected with a vacuum pump. A hole in the syringe (with piston removed) made it possible to control for suction power. The cannula was provided with a collar to control for insertion depth. The points of insertion were $3.5 \mathrm{~mm}$ behind bregma and $2.0 \mathrm{~mm}$ lateral to midline (Insertion I), and $5.0 \mathrm{~mm}$ behind bregma and $4.0 \mathrm{~mm}$ lateral to midline (Insertion II). In Insertion I, the cannula was lowered perpendicularly $3.5 \mathrm{~mm}$, and it was lowered $4.8 \mathrm{~mm}$ in Insertion II, measured from top of skull to midpoint of cannula opening. The opening of the cannula was oriented toward the hippocampal flexure for both insertions.

Bilateral TC/LEC lesions were made mechanically by means of the sharp edges of cannulas (23G, diam $0.6 \mathrm{~mm}$ ) provided with collars to control for insertion depth. The cannula to be used was mounted on a syringe. The point of insertion was $7.8 \mathrm{~mm}$ posterior to bregma and $6.7 \mathrm{~mm}$ lateral to midline. Each cannula was inserted into the brain in a position deviating $20^{\circ}$ from the vertical in the sagittal plane (tip of cannula pointing rostrally). From this posi- tion, the syringe was moved 10 times back and forth in an axis deviating about $45^{\circ}$ from the frontal plane (opening of angle pointing medially). These maneuvers were carried out in two stages, with insertion depths 6 and $8 \mathrm{~mm}$ the from the top of the skull. In this way, the distal part of the angular bundle was transected at a site corresponding approximately to the level of the rhinal fissure. The sham-operated rats were treated in the same way, except that no holes were made in the skull.

Histology. On termination of testing, the brains were removed and frozen. The brains were sectioned frontally on a $\mathrm{CO}_{2}$-freezing microtome at $30 \mu \mathrm{m}$, with every 15th section preserved in the hippocampal area and every 5th section preserved in the TC/LEC area. The sections were stained with methylene blue.

Administration of injections. A single dose of glycine $(750 \mathrm{mg} / \mathrm{kg}$ ) or physiological saline $(0.5 \mathrm{ml})$ was injected i.p. $1.5-2 \mathrm{~h}$ before Day 2 of training. Glycine (purchased from Sigma Chemical Co., St. Louis, MO) was dissolved in distilled water with $\mathrm{pH}$ adjusted to 7.4 with $\mathrm{NaOH}$.

Apparatus. Testing of simultaneous brightness discrimination was carried out in a Plexiglas cage $(56 \times 34 \times 20 \mathrm{~cm})$ previously described (Myhrer \& Nævdal, 1989). In brief, a Plexiglas wall with an opening $(10 \times 10 \mathrm{~cm})$ in the middle divided the apparatus in two equal compartments: start and goal. Three interchangeable aluminium cylinders $(3 \times 7 \mathrm{~cm})$ with a round well $(2 \times 2 \mathrm{~cm})$ in the top served as discriminanda. The cylinders were located in fixed positions (equal distance between each) along the wall opposite to the partition wall in the goal compartment. The cylinders were either natural grey (aluminium) or painted black (except for the well). The well of the positive cylinder was filled with water. The only light was a $15-\mathrm{W}$ bulb $60 \mathrm{~cm}$ above the apparatus.

Procedure. During acquisition and retention testing, the rats were deprived of water for $23.5 \mathrm{~h}$ a day. On Day 1 (Postoperative Day 8), each rat was allowed to explore the empty test apparatus for $15 \mathrm{~min}$. On Day 2, the subjects were trained to run from the start compartment into the goal compartment, in which they were rewarded with some laps of water from the well in the positive cylinder. The rats were given 10 trials, and the intertrial interval was $20 \mathrm{sec}$, during which they stayed in their home cage. On Day 3, the animals were given trials until the occurrence of five correct responses in succession. Because the task is easily learned, learning criterion was set low to avoid overlearning. Thirteen days after learning criterion had been reached, the animals were tested for retention of the discrimination task. Testing was terminated when the previous criterion was reached. Number of trials to criterion, and number and type of errors to criterion were recorded. In order to drink or investigate whether the well in a cylinder contained water, the rats had to stand on their hindlegs with at least one forepaw on top of the cylinder. Error response was scored when a negative cylinder was mounted and found empty of water (e.g., licking the empty well). Approaching or investigating negative cylinders (except the well) was not scored as an error. The positive cylinder was either black or gray, and the two cylinders of opposite color were negative. The position of the positive cylinder (left, middle, right) was changed in a prearranged randomized order. One set of randomized positions was used on Day 1 of acquisition, and another set was used on Day 2 and on retention testing. A counterbalanced paradigm was followed in which half of the subjects were trained with the black cylinder as positive and the other half with the gray cylinder as positive.

During the initial phase of learning this task, rats frequently put their snout close to negative cylinders and then leave. Because olfactory cues are of no guidance in this respect, the rats most likely respond to the color. An approach to the positive cylinder is immediately followed by rearing and drinking from the well. As training proceeds, rats gradually cease approaching negative cylinders 




Figure 1. Reconstruction of brain sections showing typical locations of hippocampal and TC/LEC lesions.

and head for the positive cylinder when entering the goal compartment. It is not likely that they change their learning strategy at this stage of training by addressing the positive cylinder because of its odd appearance (one positive vs. two negative cylinders), since approaching negative cylinders is seen now and then.
Statistics. Statistical overall analyses were made with a KruskalWallis one-way analysis of variance (ANOVA), and group comparisons were made with a two-tailed Mann-Whitney $U$ test. Computations of $U$ tests were carried out with the Minitab system, a statistical software program (Mintab, Inc.).

\section{Results}

Histology. The hippocampal lesions constituted about two thirds of this structure (see Figure 1). Neocortical or thalamic areas were only slightly affected in some animals, and the lesions rarely extended below the level of the hippocampal flexure. About two thirds of the white matter between TC and LEC were damaged (see Figure 1). The combined lesions appeared similar for both glycine- and saline-treated animals.

Reactions to injections. The injection of glycine caused an immediate, but temporary (1-2 min), paralysis of the hindlimbs. This reaction was probably associated with activation of inhibitory glycine receptors in the brain stem and spinal cord.

Behavior. Nonparametric statistics were used because of the uneven distribution of data. The glycine-treated animals appeared indistinguishable from the control rats (see Figure 2). A Kruskal-Wallis one-way ANOVA confirmed a significant treatment effect in errors on Day 1 of acquisition $[H(2)=14.16, p<.001]$. Group comparisons with a Mann-Whitney $U$ test showed that the Hipp + TC/LEC + Sal group made significantly more errors than did both the control group and the Hipp + TC/LEC + Gly group $(p<.01)$. An ANOVA also revealed reliable differences among the groups in errors on Day $2[H(2)=$ $11.69, p<.01]$. The saline-treated group made significantly more errors than did the control and glycine-treated groups $(p<.01)$. A reliable overall effect in trials to criterion was seen as well $[H(2)=11.88, p<.01]$. The Hipp + TC/LEC + Sal group used significantly more trials than did the control and Hipp + TC/LEC + Gly groups $(p<.01)$.

An ANOVA did not confirm a reliable effect in errors during retention. However, a significant treatment effect was seen in trials to criterion $[H(2)=8.11, p<.02]$. The saline-treated group used reliably more trials than did the control and glycine-treated groups $(p<.05)$.

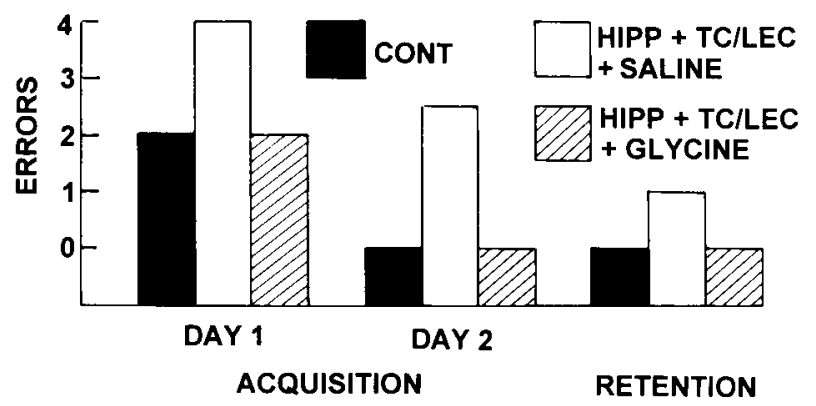

Figure 2. Median measures of error scores to criterion in Experiment 1 . 


\section{EXPERIMENT 2}

The results from Experiment 1 show that the cognitive deficits caused by combined Hipp + TC/LEC lesions are completely ameliorated by the administration of glycine. Because this lesion combination appears to enhance cognitive impairments (Myhrer \& Johannesen, 1993), the behavioral improvement obtained with glycine is somewhat surprising. However, none of the separate lesions were complete, which means that compensatory functions might have taken place in remnant glutamatergic systems. Thus, the issue of localizing the mitigating processes becomes a subject of particular interest. The putative glutamatergic systems involved in the restoration of cognitive functions may be localized either in still functional subregions of the damaged structures or in related "intact" structures. In the lesion combination, only the dorsal two thirds of the hippocampal region was destroyed, along with about two thirds of the fiber connections between TC and LEC. Because the cannula transections cannot follow the exact curvature of the rhinal fissure, the connections between a relatively small area in both TC and LEC are not accessible for denervation-in total, about one third of the fibers. Since nearly total hippocampal lesions can be achieved by means of visually guided surgery, such a specimen may contribute to highlight the issue of localizing the supporting processes initiated by pharmacological agents. In Experiment 2, the rats with extensive hippocampal lesions and controls were treated with the same procedures as those described in Experiment 1. The rats bearing radical hippocampal lesions were only expected to display impeded acquisition. The visually guided surgery implicates removal of neocortical tissue overlying the dorsal hippocampus. However, a group with cortical control lesions was not included, because the present purpose was to compare effects of glycine versus saline on total hippocampal lesions.

\section{Method}

Subjects. Twenty-four male Wistar rats, weighing 290-320 g at the time of surgery, served as subjects. They were randomly assigned to three groups: Eight rats received radical hippocampal lesions and were injected with glycine (the Hipp + Gly group), 8 received radical hippocampal lesions and were injected with saline (the Hipp + Sal group), and 8 sham-operated rats served as the control group. The rats were treated as described in Experiment 1.

Surgery. The rats were anesthetized and treated as described for Experiment 1. Bilateral hippocampal lesions were made by aspiration under an operation microscope. The sham-operated rats had only their scalps reflected. The animals were given a recovery period of 13 days before acquisition training started.

Histology, Administration of Injections, Apparatus, and Procedure. These were the same as those described in Experiment 1.

\section{Results}

Histology. The hippocampal lesions were almost complete for both saline- and glycine-treated rats (see Figures 3 and 4). A fraction of the temporal pole was left undamaged in one hemisphere in 4 rats in the Hipp + Sal group and in 3 rats in the Hipp + Gly group. In 1 rat in each of the groups, the ventral hippocampus was

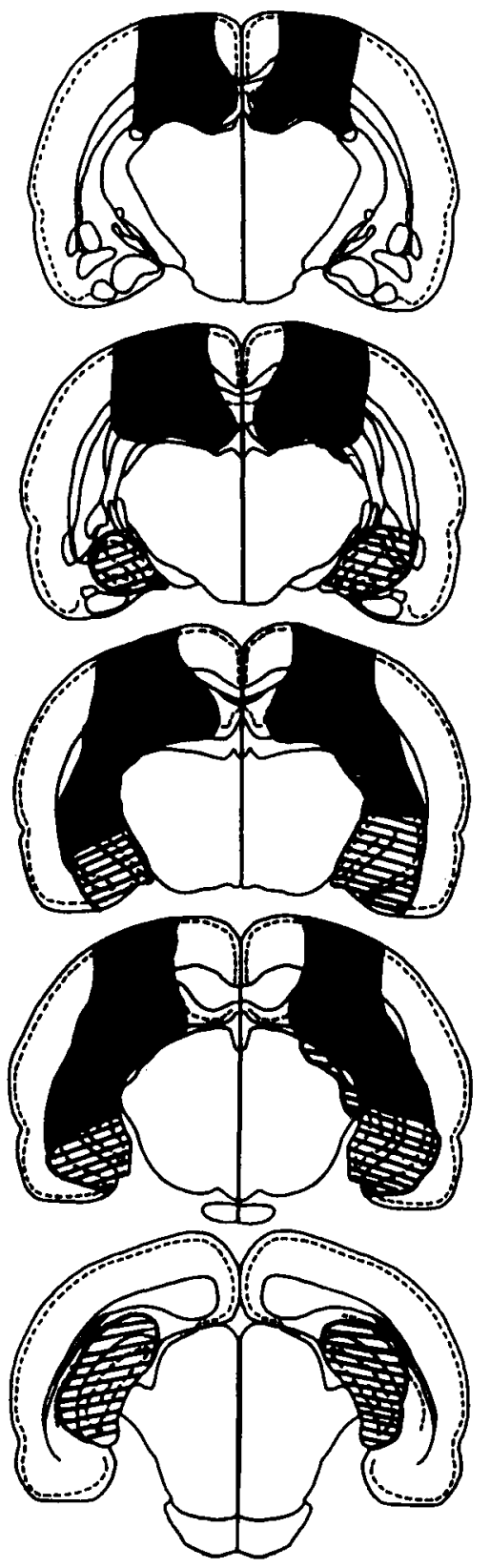

Figure 3. Reconstruction of brain sections showing location of the smallest (black) and largest (diagonal lines) hippocampal lesions.

undamaged in both hemispheres. This remnant part of the hippocampal region was probably not functionally intact, because all rats were totally deprived of the fimbria. In addition, the neocortex overlying the dorsal hippocampus had been removed. The neocortical lesions predominantly affected Areas 4, 7, 17, and 18. Unilateral damage to the dorsolateral thalamus was seen in 3 saline-treated and 2 glycine-treated animals. Bilateral or unilateral damage to the posterior amygdala and entorhinal cortex was seen in 2 saline-treated and 3 glycine-treated rats.

Behavior. Hippocampal lesions only affected the acquisition of the discrimination task (see Figure 5). A 


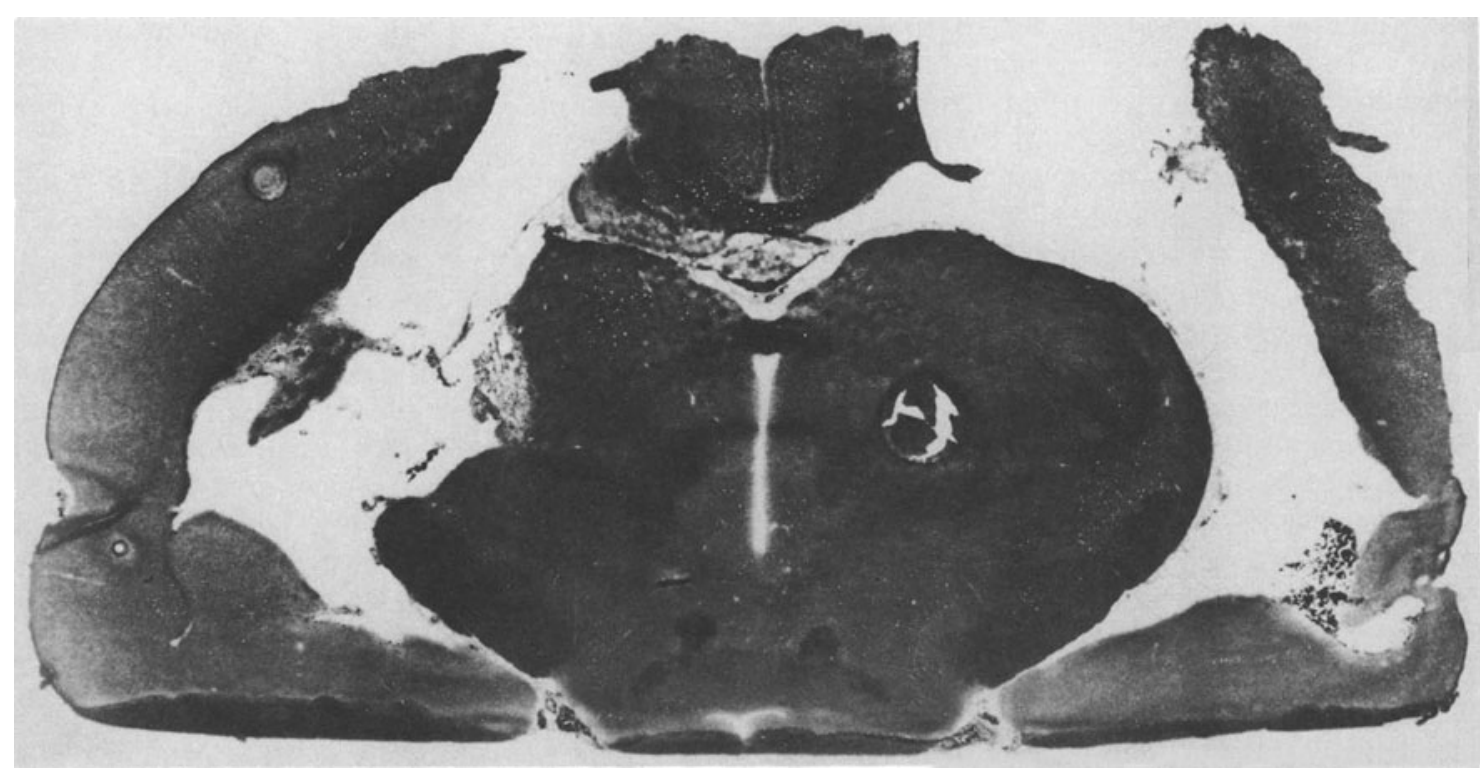

Figure 4. Photograph showing the appearance of large hippocampal lesions.

Kruskal-Wallis one-way ANOVA confirmed a significant overall effect in errors on Day 1 of acquisition $[H(2)=$ $16.13, p<.001]$. The Hipp + Sal group made reliably more errors than did the control group and the Hipp + Gly group $(p<.005)$. The latter groups did not differ significantly from one another. An ANOVA also revealed significant differences in errors on Day $2[H(2)=8.58$, $p<.02]$. The Hipp + Sal group made reliably more errors than did the control group $(p<02)$. The difference between the Hipp + Sal and Hipp + Gly groups approached statistical significance $(p=.052)$. The control and Hipp + Gly groups were not significantly at variance. A significant treatment effect also emerged in terms of trials to criterion $[H(2)=7.40, p<.05]$. The Hipp + Sal group used more trials than did the control group, but not more than did the Hipp + Gly group. The control and Hipp + Gly groups did not differ reliably from one another.

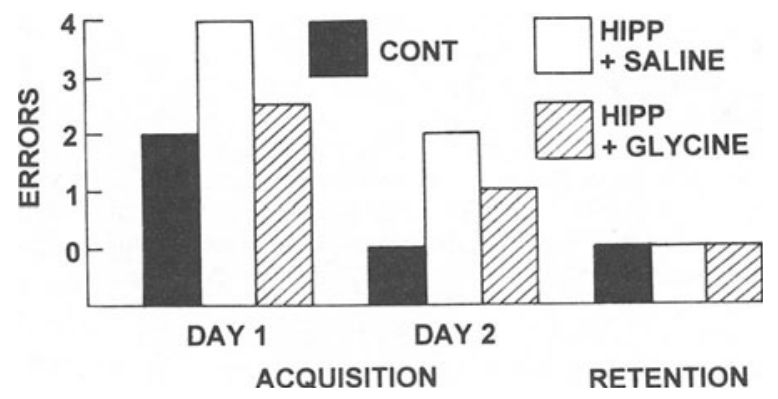

Figure 5. Median measures of error scores to criterion in Experiment 2.

\section{GENERAL DISCUSSION}

The results from the present study show that the acquisition and retention deficits produced by combined hippocampal and TC/LEC lesions are completely mitigated by administration of glycine (Experiment 1). Furthermore, the severe acquisition deficit seen to be caused by radical hippocampal lesions was attenuated, but not completely ameliorated, by glycine (Experiment 2 ).

It is difficult to attribute the differences seen among the lesioned groups to factors other than the agents injected. The location and extent for the combined lesions in Experiment 1 and the single lesion in Experiment 2 were very similar across saline- and glycine-treated groups. It might be argued that the neocortical damage caused by the radical hippocampal lesions prevented full recovery of the acquisition deficit in rats injected with glycine. This possibility cannot be entirely ruled out, but rats with neocortical lesions corresponding to those seen in this study are unimpaired in acquiring the present discrimination task (Myhrer, 1992). Moreover, the effects obtained with glycine are probably not attributable to peripheral adrenergic mechanisms, because the blockade of sympathetic terminal release has been shown not to prevent the mitigating effects of glycine (Myhrer \& Paulsen, 1992).

It might have been expected that nearly total hippocampal lesions would cause a more severe acquisition deficit than would subtotal Hipp + TC/LEC lesions. It has been shown, however, that acquisition is even more impaired when incomplete hippocampal lesions are combined with LEC or TC/LEC lesions than when obtained with corresponding hippocampal lesions alone (Myhrer \& Johannesen, 1993). Selective LEC lesions do not im- 
pede acquisition, whereas TC/LEC lesions only impede acquisition on Day 2 of training, suggesting that the deficit is of mnemonic nature (Myhrer, 1992). Subtotal hippocampal lesions combined with LEC or TC/LEC lesions seem to equalize the acquisition deficit that has been seen to follow extensive hippocampal lesions.

Glycine administered to rats with radical hippocampal lesions resulted only in some improvement of the learning function and not in complete restoration. The performance in terms of errors on Day 2 of acquisition was not significantly better in the Hipp + Gly group than in the Hipp + Sal group, although the difference approached a reliable level $(p=.052)$. This result suggests that, in the case of complete lesions, administration of glutamatergic agonists may have limited impact on recovery. Because the hippocampal region was removed, the ameliorating processes probably took place in functionally related structures. Potential candidates may predominantly have been glutamatergic systems in the temporal-entorhinal areas and, perhaps, in the septum. The septal and entorhinal areas have major projections to the hippocampal region. Retrograde degeneration in these structures probably contributes to the hippocampal lesions effect. Although the present study only provides data for the hippocampal region, it might be possible to suggest some general principles for the restoration of impaired functions. In the case of subtotal lesions, functionally remnant subregions may primarily include the compensatory processes. It is also possible that agent-induced auxillary processes in remnant subregions act in concert with compensatory process in functionally related structures. In the case of total lesions, supporting processes probably take place predominantly in neighboring and/or functionally related structures provided that they contain glutamatergic systems.

The findings that glycine counteracted memory impairment may suggest that this agent interferes more with functional mechanisms than with pharmacological ones. Although it remains to be demonstrated, it is most likely that glycine made NMDA receptors more sensitive to endogeneous glutamate. Glycine has been shown to potentiate NMDA responses enormously in cultured mouse brain neurons (Johnson \& Ascher, 1987). Because beneficial effects of glycine were seen 2 weeks after injection in this study, there are reasons to believe that glycine acted via LTP. It may be assumed that vivid LTP activity is involved in the mechanisms underlying restoration of impaired functions when induced by glutamatergic agonists.

Glycine probably does not have a general enhancing effect on memory, but it may modulate memory during certain circumstances. In a previous study, it was shown that glycine administered midway between acquisition and retention did not improve memory, whereas glycine given either just prior or just after acquisition, and just prior to retrieval, improved retention in rats with TC/LEC lesions (Myhrer et al., 1993). It is possible that memory engrams associated with a specific task have to be partic- ularly activated during the influence of agonists. Glutamatergic agonists are presumably useful for neural activity during specific encoding and retrieval of sensory information.

Some groups of patients may derive profit from treatment with glutamatergic agonists. Examination of samples of cortical tissue that have been removed surgically for diagnostic purposes from patients with Alzheimer's disease reveals loss of corticocortical association fibers and reduced content of glutamate (Francis, Pangalos, \& Bowen, 1992). These findings have led the latter authors to suggest treatment of the disease with the partial glutamatergic agonist D-cycloserine to compensate for the declining cognitive capability. Because glycine is a very potent agonist that may run the risk of causing further damage by excitotoxic effects, partial agonists may represent an appropriate alternative. It should be emphasized in this respect that glycine is only considered as a convenient pilot agent, and that further experimentation may lead to the development of agonists that will more properly serve the purpose in individual cases.

The hippocampal region is an early and critical site of pathology both in Alzheimer's disease and in Down's syndrome (Stoll, Balbo, Ault, Rapoport, \& Fine, 1993). The hippocampus is also severely destroyed in amnesic patients with medial temporal lobe resections. The present finding that glycine produced a marked improvement of the learning ability in rats with radical hippocampal lesions may suggest that individuals with Down's syndrome or temporal injuries may profit from treatments that enhance glutamatergic neurotransmission.

\section{REFERENCES}

Deutsch, S. I., \& Morihisa, J. M. (1988). Glutamatergic abnormalities in Alzheimer's disease and a rationale for clinical trials with Lglutamate. Clinical Neuropharmacology, 11, 18-35.

FoNNUM, F. (1984). Glutamate: A neurotransmitter in mammalian brain Journal of Neurochemistry, 42, 1-11.

Francis, P. T., Pangalos, M. N., \& Bowen, D. M. (1992). Animal and drug modelling for Alzheimer synaptic pathology. Progress in Neurobiology, 39, 517-545.

Johnson, J. W., \& AsCHER, P. (1987). Glycine potentiates the NMDA response in cultured mouse brain neurons. Nature, 325, 529-531.

KöHLER, C. (1986). Cytochemical architecture of the entorhinal area. In R. Schwarcz \& Y. Ben-Ari (Eds.), Excitatory amino acids and epilepsy (pp. 83-98). New York and London: Plenum.

MYhRER, T. (1992). Selective lesions in the temporal-hippocampal region of the rat: Effects on acquisition and retention of a visual discrimination task. Behavioral \& Neural Biology, 58, 8-15.

MYHrer, T. (1993). Animal models of Alzheimer's disease: Glutamatergic denervation as an alternative approach to cholinergic denervation. Neuroscience \& Biobehavioral Reviews, 17, 195-202.

Myhrer, T., IVerSEN, E. G., \& FonNum, F. (1989). Impaired reference memory and reduced glutamergic activity in rats with temporoentorhinal connections disrupted. Experimental Brain Research, 77, 499-506.

MYHRER, T., \& JOHANNESEN, T. S. (1993). Learning and retention of a visual discrimination task in rats with various combinations of lesions in the temporal-hippocampal region. Manuscript submitted for publication. 
Myhrer, T., Johannesen, T. S., \& Spikkerud, E. (1993). Restoration of mnemonic function in rats with glutamergic temporal systems disrupted: Dose and time of glycine injections. Pharmacology, Biochemistry \& Behavior, 45, 519-525.

MyHRER, T., \& N\&VDAL, G. A. (1989). The temporal-hippocampal region and retention: The role of temporo-entorhinal connections in rats. Scandinavian Journal of Psychology, 30, 72-80.

Myhrer, T., \& Paulsen, R. E. (1992). Memory dysfunction following disruption of glutamergic systems in the temporal region of the rat: Effects of agonistic amino acids. Brain Research, 599, 345-352.

SQUIRE, L. R. (1992). Memory and the hippocampus: A synthesis from findings with rats, monkeys and humans. Psychological Review, 99, 195-231.
Stoll, J., Balbo, A., Ault, B., Rapoport, S. I., \& Fine, A. (1993). Long-term transplants of mouse trisomy 16 hippocampal neurons, a model for Down's syndrome, do not develop Alzheimer's disease neuropathology. Brain Research, 610, 295-304.

Toth, E., \& LAJTHA, A. (1981). Elevation of cerebral levels of nonessential amino acids in vivo by administration of large doses. Neurochemical Research, 6, 1309-1317.

(Manuscript received July 20, 1993; revision accepted for publication October 20,1993 .) 